\title{
Biofeedback as single first-line treatment for non-neuropathic dysfunctional voiding children with diurnal enuresis
}

Fahad Alyami; Tamer Ewida; Hamdan Alhazmi; Mahmoud Trbay; Mostafa Arafa; Moina Tahir; Khalid Fouda Neel

Division of Urology, Department of Surgery, College of Medicine, King Saud University Medical City, King Saud University, Riyadh, Saudi Arabia

Acknowledgment: We would like to thank the College of Medicine Research Center (CMRC) Deanship for Scientific research, King Saud University for supporting this work.

Cite as: Can Urol Assoc J 2018 July 24; Epub ahead of print. http://dx.doi.org/10.5489/cuaj.5209

\section{Published online July 24, 2018}

\section{$* * *$}

\section{Abstract}

Introduction: Non-neurogenic dysfunctional voiding (NDV) accounts for a significant portion of pediatric urology outpatient clinic visits. Biofeedback (BF) is a promising, non-invasive modality for treating children with DV and daytime wetting. Our objective was to investigate BF's efficacy as a single first-line treatment for children with NDV and diurnal enuresis.

Methods: A retrospective cohort study was conducted with a total of 61 consecutive patient records from January 2009 to March 2016. All children with NDV who had BF as first-line treatment were included. Full urological histories, physical examinations, dysfunctional voiding symptom score (DVSS), urine analysis, ultrasound (US), and uroflowmetry (UFM), and electromyogram (EMG) were performed and recorded for all patients before and after finishing the last BF cycle. The patient's satisfaction scale was also obtained.

Results: The mean age was $10 \pm 2.6$ years. Most patients (80.3\%) were females. The presenting symptoms were diurnal enuresis, urinary tract infections, and voiding discomfort in 52 (85.2\%), 16 (26.2\%), and 38 (62.3\%) patients, respectively. Six months after the last BF cycle, there was a statistically significant objective improvement in US and UFM+EMG findings with the disappearance of EMG signals in 40 of 61 (65.5\%) patients. There was also a significant subjective symptomatic improvement, as the mean DVSS had decreased from 14 to 7.9 ( $\mathrm{p}=0.003)$. Fortyseven patients (77\%) were satisfied, while only eight (13.1\%) were not.

Conclusions: BF is considered a potentially effective, single first-line treatment modality for children with DV and diurnal enuresis. Long-term outcome assessments are needed to assess the children's compliance and symptom recurrence. 


\section{Introduction}

Pediatric dysfunction voiding (DV) accounts for a significant portion of outpatient pediatric urologist clinics visits. DV can have either a neurogenic or non-neurogenic etiology. ${ }^{1,2}$ A nonneuropathic dysfunctional voiding (NDV) child could present with recurrent urinary tract infections (UTIs), diurnal enuresis, and/or voiding discomfort. ${ }^{3}$ Classical NDV management starts with behavioral therapy. If behavior therapy fails, the other treatment options could be one or more of some other treatments: 1) anticholinergics; 2) alpha-adrenergic blockers; 3) intra-vesicular botulinum toxin injections; and/or 4) biofeedback (BF). ${ }^{4}$

$\mathrm{BF}$ is a non-invasive, promising modality for treatment of children with DV and daytime enuresis, to help these children to be more aware about their normal voiding cycle, and to help them relax their pelvic floor muscles voluntary during voiding. ${ }^{5,6}$ The use of computer-based audio and visual games that are used to maintain the interest of a child in conjunction with a biofeedback therapist who acts as a coach and gives hints on how to improve the child's performance make it an attractive treatment option for children with DV. ${ }^{5,6}$

BF by itself can't cure NDV; BF depends mainly on the children and their families' motivation and their dedication to the program in addition to the standard behavioral therapy. The children must commit themselves to practice pelvic floor muscles exercises daily, and they must accept their responsibility for maintaining their own health.

In the literature, the studies that address using BF as the first, single line of treatment for NDV are scare. Our objective was to investigate BF's efficacy as a single first-line treatment for NDV children with diurnal enuresis in conjunction with the standard behavioral modifications.

\section{Methods}

This is a retrospective cohort study, in which data were obtained between January 2009 and March 2016 in our institute from a prospectively maintained database. This study was approved by our institutional research ethics board. All children $>5$ years of age with abnormal voiding patterns or diurnal enuresis with NDV as documented by electromyogram (EMG) signals in uroflowmetry (UFM)+EMG were enrolled in the study. Patients with several criteria were excluded from the study: 1.) neurological abnormality; 2.) patients who had undergone surgical intervention or received medical treatment for management of their NDV; and 3.) patients unable to follow the biofeedback protocol because of his/her low mental status or whose place of residence was too far and could not get to the BF sessions.

All patients were exposed to thorough evaluation before starting the BF sessions (full urological history and physical examination). A subjective assessment of the DV symptoms was conducted by self-assessment dysfunctional voiding symptom score (DVSS). Urine analyses (UA), ultrasound (US), and UFM+EMG with assessment of post-voiding residual (PVR) were conducted for all patients before starting the BF cycle.

Each BF cycle was composed of six sessions with one week apart between a session. The initial assessment and BF protocol planning were conducted based on the first session. After 
finishing each session, the BF therapist instructed the child and his/her parents about home pelvic floor muscle exercises, timed voiding, voiding position, self-cleaning, and dietary control. Their home compliance was recorded. At the end of the BF cycle, each patient was reassessed subjectively by DVSS and patients' satisfaction scale forms that had been filled out by the child or his/her family. Six months later all of the patients were reassessed objectively by UA, US, and UFM+EMG with PVR assessment.

$\mathrm{BF}$ cycles were repeated once or twice if there was unsatisfactory objective improvement in the patient's clinical condition and whether the patient's family was motivated to do more cycles in order to improve their child's performance.

Data with different variables were illustrated by simple frequency distribution, t-test, and paired t-test, which were used to compare quantitative data. A z-score test was also used for comparing percentages in both groups (such as before and after BF). The level of significance was set at $95 \%$.

\section{Results}

A total of 61 patients with average age of $10 \pm 2.6$ years (range 5-7 years). Most of our patients were female 49 (80.3\%). The presenting symptoms included daytime wetting (52, 85.2\%), recurrent UTIs (16, 26.2\%), and/or voiding discomfort or straining during voiding (38, 62.3\%) (figure 1).

All patients had hyperactive pelvic floor muscles as seen on the EMGs. It is also worth mentioning that 24 (39.3\%) patients received one BF cycle, 31(50.8\%) patients received two cycles, and only six (9.8\%) patients received three BF cycles. Only 30 patients were compliant with home instructions and pelvic floor muscles exercises. Subjective and objective parameters before and after BF cycles are illustrated in Tables 1 and 2.

There was a statistically significant symptomatic improvement in diurnal enuresis, urinary tract infections, and voiding discomfort. The mean DVSS decreased significantly from $14 \pm 4.9$ to $7.9 \pm 5(\mathrm{p}=0.003)$ as shown in Table 1.

After assessing the objective parameters including hydronephrosis/renal parameters, postvoiding residual as shown by US, UFM curve shape, and Q-average $\left(\mathrm{Q}_{\mathrm{ave}}\right)$ in UFM, it was observed that all parameters improved significantly (Table 2) with disappearance of EMG signals in $40(65.5 \%)$ patients ( $\mathrm{p}=0.000)$ as shown in Table 2.

When we followed the patients for 6 months at least we found 30 (49\%) stopped doing the biofeedback and the reported recurrence of symptoms was $47 \%$.

After examining at the overall patient's satisfaction after BF sessions, it was noted that 47 (77\%) patients were satisfied, six (9.8\%) were neutral, and eight (13.1\%) were not satisfied. In our series, we did not encounter any adverse events related to use of BF or urotherapy.

\section{Discussion}

In the literature, BF use in addition to the standard behavioral modifications in children with NDV showed variable results. Some studies showed promising results and others presented discouraging 
results. ${ }^{7}$ A third of the children with NDV showed unsatisfactory results if urotherapy was used alone. ${ }^{8}$ In the current study, we evaluated BF's effectiveness as a first-line treatment for NDV in children with diurnal urinary enuresis.

Two systematic reviews were done looking at BF's effect on daytime wetting in children, and they showed contradicting results. Fazeli et al. did a systematic review and meta-analysis of randomized controlled trials looking at BF's effects on NDV and found the overall proportion of cases with resolved incontinence at six months was similar in the biofeedback and control groups. They also found no significant differences in mean maximum urinary flow rate or likelihood of UTIs. $^{9}$

However, Desantis et al. showed significant improvement by $83 \%$ and $80 \%$ in UTI and daytime incontinence, respectively, as a result of BF for NDV. ${ }^{10}$

We report a statistically significant symptomatic improvement with diurnal enuresis and urinary tract infection after BF (P values 0.01 and 0.03 , respectively). The mean DVSS decreased significantly from $14 \pm 4.9$ to $7.9 \pm 5(\mathrm{p}=0.003)$.

Our study found a statically significant objective improvement in the mean $\mathrm{Q}_{\text {ave }}$ (decreased from 8.8 to $12.9 \mathrm{ml} / \mathrm{sec}$ ) and mean PVR (reduced by 50\%, $\mathrm{p}=0.000$ ).

Nelson et al. reported significant improvements in UFM parameters in children treated with BF for DV with an increase in peak flow and average flow rate and a significant decrease in PVR (Qmax 21.0 to $24.8 \mathrm{~mL} / \mathrm{s}$; Q Qve, 9.1 to12.0 mL/s; and PVR, 75 to $34 \mathrm{~mL})(11)$.

Kajbafzadeh et al. found that_bladder capacity and voided volume did not significantly improve post BF in kids with DV, but the PVR and voiding time decreased considerably, while maximum and average urine flow increased significantly. ${ }^{2}$

Desantis et al. showed PVR improvement ranging from 26 to $99 \mathrm{ml}$ and $\mathrm{Q}_{\max }$ and $\mathrm{Q}_{\text {ave }}$ increased from 3.8 to $4.7 \mathrm{ml} / \mathrm{s}$ and $3.1 \mathrm{ml} / \mathrm{s}$, respectively. ${ }^{10}$

We admit for such treatment option compliance is of a major concern as it depends on the kid motivation. To overcome the compliance issue our urotherapist contacts the family of the children on a regular basis to follow up the progress during treatment in addition to the regular clinic visits.

Biofeedback for NDV has slowly gained popularity and there is no standardized protocol and no clear criteria to recruit patients who would benefit from this therapy. Also the parameters of improvements are very variable and mainly subjective which make it harder to assess in children. In this paper we propose BF as a valid option for patients with NDV and we recommend individualizing the treatment based on the patients condition, presentation, age and motivation. Known that there will be always bias of choosing the treatment option according to the physician preference, available resources and patient factors.

We acknowledge that the retrospective design of our study and the small number of patients are the main limitations of our study. We are working on the long-term studies in a prospective manner for these children. 


\section{Conclusion}

$\mathrm{BF}$ is a reliable and feasible first line treatment modality for children with non-neurogenic dysfunctional voiding and diurnal enuresis. Prospective studies on long-term outcomes using this approach are necessary to support our data. 


\section{References}

1) Cooper CS Voiding dysfunction. Accessed at www.emedicine.medscape.com/article/101698-overview

2) Kajbafzadeh AM, Sharifi-Rad L, Ghahestani SM, et al. Animated biofeedback:an ideal treatment for children with dysfunctional elimination syndrome. J Urol 2011; 186 (6):237984.

3) Järvelin MR, Vikeväinen-Tervonen L, Moilanen I, et al. Enuresis in seven-year-old children. Acta Paediatr Scand 1988;77:148.

4) Sillén U, Hellström AL. Pragmatic approach to the evaluation and management of nonneuropathicdaytime voiding disorders. In: Pediatric Urology, 2nd ed. Edited by JP Gearhart, RC Rink and PDE Mouriquand. Philadelphia: Saunders Elsevier 2010; chapt 28, p 366.

5) Koenig JF, Mckenna PH. Biofeedback therapy for dysfunctional voiding in children. Curr Urol Resp 2011;12(2):144-52.

6) Combs AJ, Glassberg AB, Gerdes D, et al. Biofeedback therapy for children with DV. J Urol 1998;52(2):312-15.

7) VanGool JD, de Jong TP, Winkler-Seinstra P, et al. Multi-center randomized controlled trial of cognitive treatment, placebo, oxybutynin, bladder training, and pelvic floor training in children with functional urinary incontinence. Neurourol Urodyn 2014;33:482.

8) Lordêlo P, Benevides I, Kerner EG, et al. Treatment of nonmonosymptomatic nocturnal enuresis by transcutaneous parasacral electrical nerve stimulation. J Pediatr Urol 2010;6:486-89.

9) Mir Sohail Fazeli, Yiqun Lin, Kourosh Afshar et al. Biofeedback for Nonneuropathic Daytime Voiding Disorder in Children: A Systematic Review and Meta-Analysis of Randomized Controlled Trials. J Urol. 2015 Jan;193(1):274-9.

10) Desantis DJ , Leonard MP , Guerra LA, et al .Effectiveness of biofeedback for dysfunctional elimination syndrome in pediatrics: A systematic review. J Ped Urol (2011) 7, 342e348.

11) Nelson JD, Cooper CS, Boyt MA, et al. Improved uroflow parameters and post-void residual following biofeedback therapy in pediatric patients with dysfunctional voiding does not correspond to outcome. J Urol 2004;172:1653-56; discussion 1656. 
Figures and Tables

Fig. 1. Patient demographics. UTI: urinary tract infection.

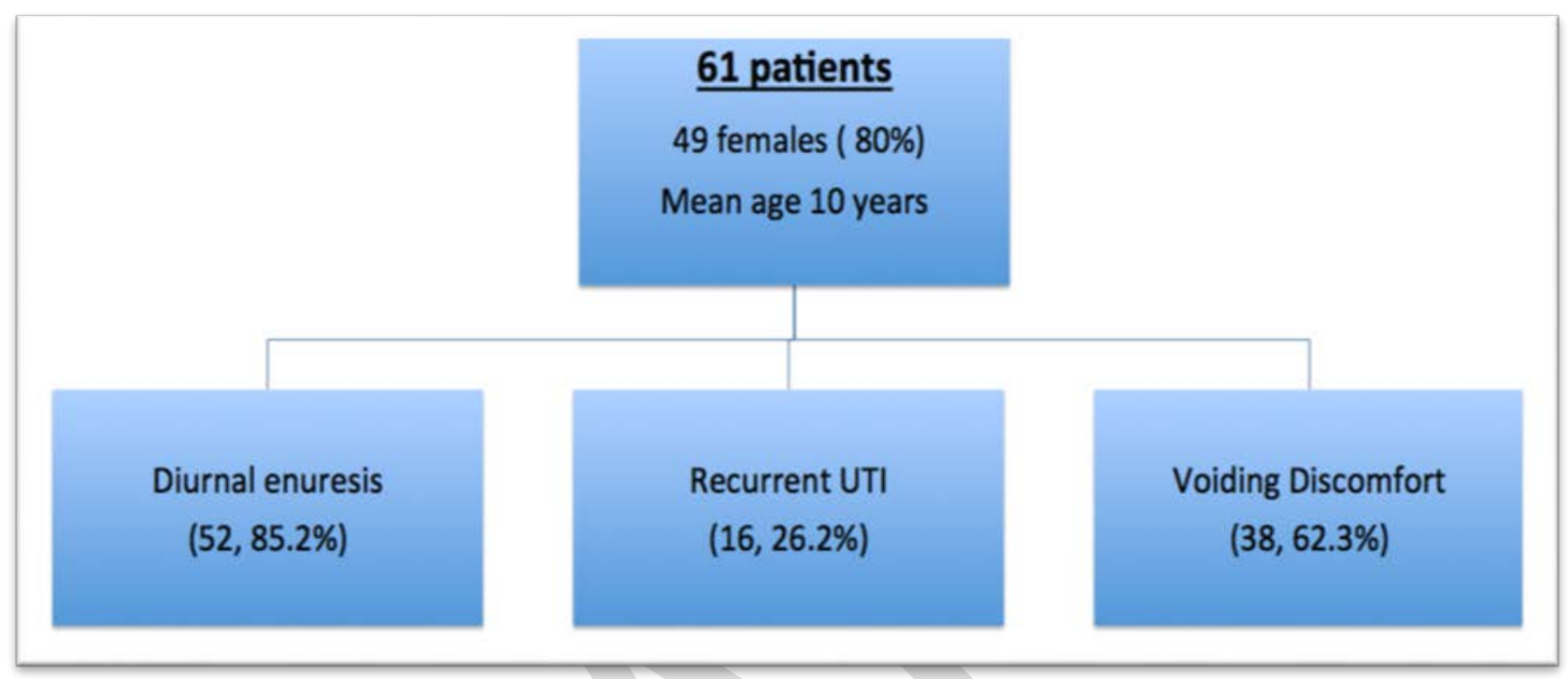

Table 1. Subjective parameters used for comparison of BF efficacy

\begin{tabular}{|l|c|c|c|c|}
\hline Symptoms & $\begin{array}{c}\text { Before BF NO } \\
(\mathbf{\%})\end{array}$ & $\begin{array}{c}\text { After BF } \\
\text { NO (\%) }\end{array}$ & Z score & p \\
\hline DE & $52(85.2 \%)$ & $21(34.4 \%)$ & 5.7 & 0.01 \\
UTI & $16(26.2 \%)$ & $8(13.1 \%)$ & 1.82 & 0.03 \\
\hline & Mean \pm SD & Mean \pm SD & Paired t test & p \\
\hline DVSS & $14 \pm 4.9$ & $7.9 \pm 5$ & 8.6 & 0.003 \\
& & & & \\
\hline
\end{tabular}

BF: biofeedback; DE: diurnal enuresis; DVSS: dysfunctional voiding symptom score; UTI: urinary tract infection. 


\begin{tabular}{|l|c|c|c|c|}
\hline \multicolumn{7}{|l|}{ Table 2. Objective parameters used for comparison of BF efficacy } \\
\hline & $\begin{array}{c}\text { Before BF NO } \\
(\%)\end{array}$ & $\begin{array}{c}\text { After BF } \\
\text { NO (\%) }\end{array}$ & Z score & p \\
\hline Hydronephrosis (US) & $45(36.8 \%)$ & $18(14.7 \%)$ & 3.9 & 0.000 \\
Obstructed UFM curve & $31(50.8 \%)$ & $8(13.1 \%)$ & 4.46 & 0.000 \\
Bell-shape UFM curve & $17(27.9 \%)$ & $45(73.8 \%)$ & 5.07 & 0.000 \\
EMG signals in & $61(100 \%)$ & $21(34 \%)$ & 7.7 & 0.000 \\
UFM+EMG & & & & \\
\hline & Mean \pm SD & Mean \pm SD & Paired t test & p \\
\hline PVR (ml) (US) & $77 \pm 58.6$ & $46.6 \pm 36.6$ & 5.7 & 0.000 \\
Q average (ml/sec) & $8.8 \pm 3.6$ & $12.9 \pm 5.5$ & 6.7 & 0.000 \\
\hline
\end{tabular}

BF: biofeedback; EMG: electromyogram; PVR: post-void residual; SD: standard deviation; UFM: uroflowmetry; US: ultrasound. 